\title{
ANÁLISIS DE LA PERCEPCIÓN DE LOS RIESGOS NATURALES EN LA UNIVERSIDAD DE ALICANTE
}

\author{
Rodrigo Rudge Ramos Ribeiro ${ }^{\mathrm{a}}$, Jorge Olcina Cantos $^{\mathrm{b}}$ y Sergio Molina Palacios ${ }^{\mathrm{c}}$ \\ Instituto Multidisciplinar para el Estudio del Medio "Ramón Margalef". Universidad de Alicante ${ }^{a}$ \\ Instituto Interuniversitario de Geografía. Universidad de Alicante ${ }^{b}$ \\ Departamento de Ciencias del Mar y Biología Aplicada. Universidad de Alicante ${ }^{c}$
}

\section{RESUMEN}

Los estudios sobre percepción de riesgos intentan analizar las relaciones afectivas y éticas que una comunidad establece con el ambiente en que vive. Las percepciones ambientales son entendidas como la forma en que cada persona aprecia y valora su entorno. El presente artículo tiene como objetivo analizar la percepción de riesgos naturales en los miembros de la comunidad académica de la Universidad de Alicante. Para evaluar la percepción se aplicaron encuestas. Han sido contestadas 80 encuestas, todas por medio electrónico. Los resultados indican que la percepción de las principales amenazas por fenómenos naturales son: las inundaciones, las sequías y los incendios forestales. Se concluye resaltando la importancia de trabajos que aporten información sobre la percepción ambiental, con el fin de hacer más eficiente la aplicación de políticas ambientales.

Palabras clave: riesgos naturales, percepción de riesgos, inundaciones, Alicante.

\begin{abstract}
Analysis of the perception of natural hazards by the members of the University of Alicante

The studies about risk perception try to investigate the relationship affective and ethic that the community established with the environment where they are living. The environmental perception is understood as the way in which each person appreciates and values the environment. This article had as objective to analyse the perception of natural hazards in the members of the academic community of the University of Alicante. To evaluate the perception surveys were applied. The result shows that the perceptions of the main natural phenomena where the respondents live are: floods, droughts and forest fires. The conclusion highlights the importance of work to provide information about environmental perception, in order to streamline the implementation of environmental politics.
\end{abstract}

Key words: natural risks, risk perception, flood, natural disaster, Alicante.

\section{INTRODUCCIÓN}

Los impactos de los riesgos naturales son importantes en el contexto del desarrollo sostenible de la sociedad. Los efectos de estos problemas ambientales han surgido en el mundo de varias maneras, tales como el impacto de inundaciones que muestran la importancia de este asunto. Siendo muy importante la participación de la población en las acciones de gestión del riesgo (Coelho et al., 2004). La percepción del riesgo es un componente del análisis del riesgo; las decisiones en su gestión están condicionadas por distintos factores, donde se incluye la percepción social del riesgo (Almeida, 2004).

El litoral alicantino constituye una zona residencial y de actividad turística del Mediterráneo español. Durante las tres últimas décadas, las lluvias torrenciales y las inundaciones han provocado cuantiosas pérdidas materiales y víctimas humanas. Ello se debe al incremento de la vulnerabilidad ante el peligro

Contacto: Rodrigo Rudge Ramos Ribeiro: rrr35@alu.ua.es; Jorge Olcina Cantos: jorge.olcina@ua.es; Sergio Molina Palacios: sergio.molina@gcloud.ua.es 
natural de precipitaciones de fuerte intensidad horaria, como consecuencia del aumento en la exposición física a los mismos. La existencia de lluvias de fuerte intensidad horaria en Alicante se produce en forma de aguaceros torrenciales, generalmente relacionados con el proceso de gota fría, y suelen tener consecuencias negativas en el territorio. Provocan también problemas de erosión en los suelos al entrar en funcionamiento las ramblas, que están la mayor parte del año secas. Las lluvias de fuerte intensidad se erigen como el peligro climático más importante de las tierras alicantinas. La Figura 1 y Figura 2 presentan los daños causados por inundaciones en el Campus de la Universidad de Alicante en el año de 1982.

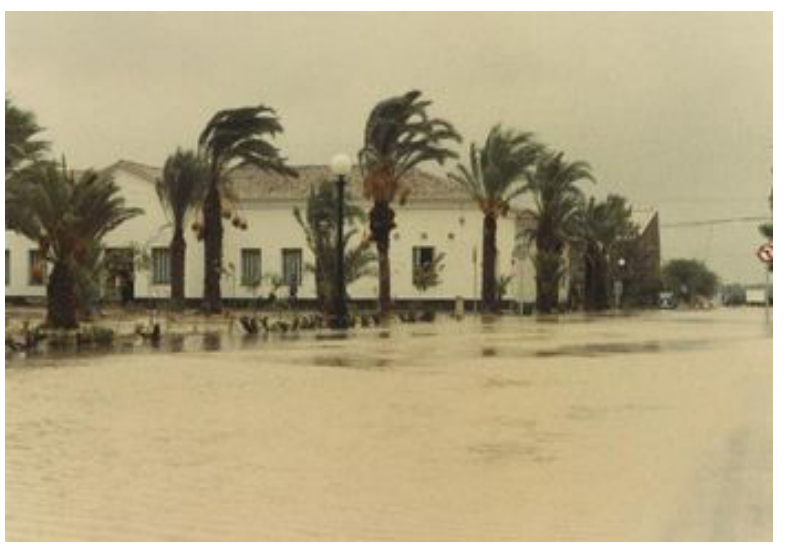

Figura 1. Inundación en el Campus de la Universidad de Alicante en octubre de 1982 (Archivo: Universidad de Alicante).

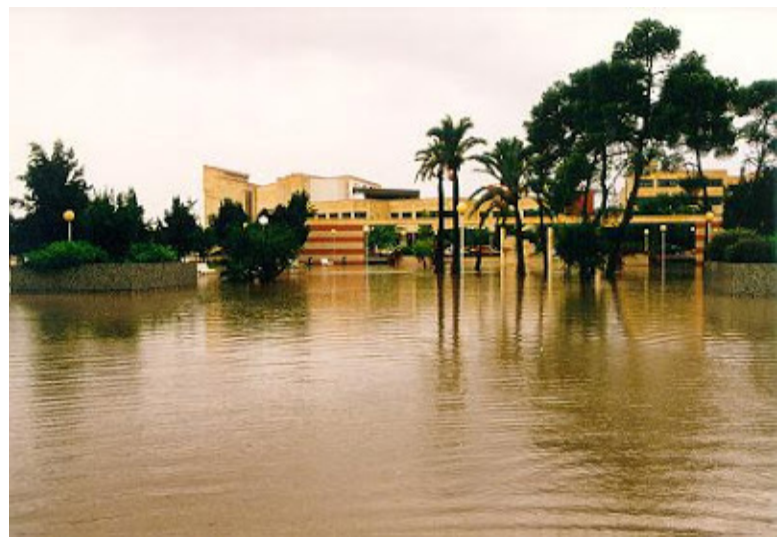

Figura 2. Inundación en el Campus de la Universidad de Alicante en octubre de 1982 (Archivo: Universidad de Alicante).

La percepción del riesgo se relaciona con una construcción de pensamiento humano, siendo un resultado social y distinto de acuerdo con los contextos experimentados por individuos o grupos, que generan múltiples interpretaciones del evento (Acosta, 2005). La percepción del riesgo es una herramienta para los servicios de protección civil y otras entidades de seguridad en los procesos de decisiones y acciones (Coelho, 2005). La perspectiva social del riesgo es, sin duda, una importante herramienta en su gestión (Weingart, 2007).

La valoración de la percepción social del riesgo de inundación requiere un análisis integrado de los dos sistemas que lo integran: natural (peligrosidad) y el humano (exposición y vulnerabilidad). Hay distintos enfoques sobre la percepción, uno de ellos es el que se refiere a la forma en que un individuo interpreta y valora los posibles efectos y peligros de un riesgo (Juan-Pérez, 2007). La percepción social de los episodios naturales otorga grado al impacto que causa un hecho natural de rango extraordinario (Olcina Cantos, 2005).

\section{MÉTODO}

Para valorar la percepción del riesgo de inundación se han elaborado encuestas dirigidas a la comunidad académica de la Universidad de Alicante. Las encuestas se realizaron en la Universidad de Alicante básicamente por dos motivos: investigar la percepción de los riesgos naturales de la comunidad académica y por ser Alicante una zona altamente expuesta a riesgos naturales como las inundaciones.

El motivo de elegir la Universidad de Alicante en este estudio es por estar en una zona de inundaciones históricas, como la inundación sufrida en el Campus de la Universidad de Alicante en octubre de 1982.

Los problemas ambientales son cada vez más importantes en el contexto de la sociedad y desempeñan un papel decisivo en el desarrollo sostenible. La justificación de las preguntas elaboradas en la encuesta son: relevancia del tema en el contexto global y en España para la sociedad y conocer la opinión de la comunidad académica en el ámbito de las estrategias educativas. Además, al existir una investigación hecha en el año de 2008 en la Universidad de Aveiro, en Portugal, sobre el tema de percepción de riesgos naturales, se optó por realizar algunas preguntas similares. 
Figura 3. Localización de la Universidad de Alicante en su contexto territorial.

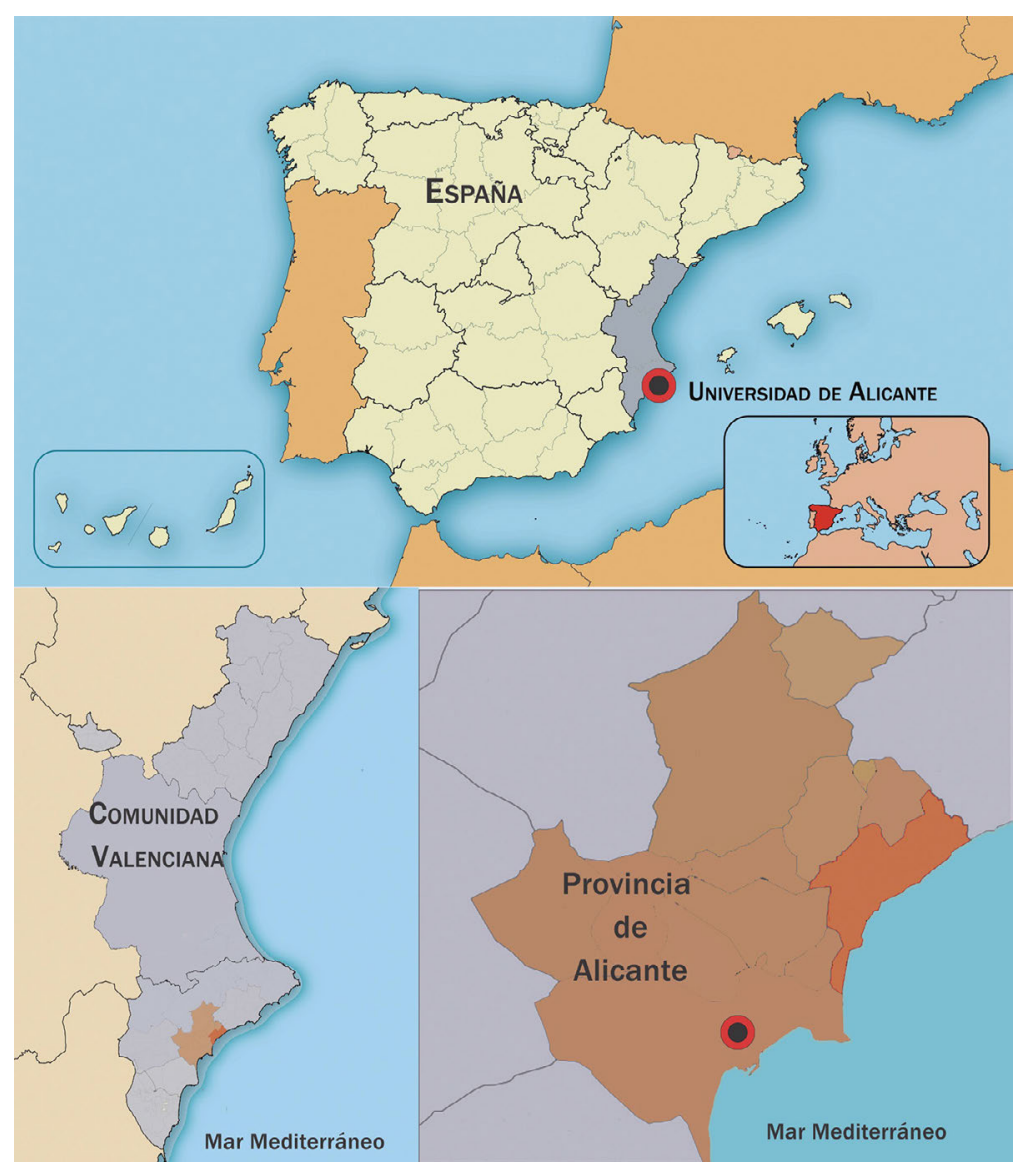

El análisis de los aspectos perceptivos del riesgo se realizó por medio de encuestas. La finalidad de estas se centra de manera sistemática y ordenada en obtener información sobre variables que intervén en la percepción de riesgos naturales. Las encuestas utilizadas intentan determinar cuál es la percepción social de los riesgos naturales. Para determinar el muestreo recomendado de las encuestas se utilizó el método del muestreo probabilístico (ecuación 1), donde fue aplicada la ecuación para determinar el tamaño de muestra (n) para una población finita:

$$
n=\frac{N \times \sigma^{2} \times\left(Z_{\alpha / 2}^{2}\right)}{(N-1) \times E^{2}+\sigma^{2}\left(Z_{\alpha / 2}^{2}\right)}
$$

Donde: $\mathrm{n}$ - tamaño de la muestra,

$\mathrm{N}$ - nivel de confianza,

$\sigma-$ varianza,

E - margen de error,

Z - intervalo de confianza.

Se realizó una fase piloto previa en Universidad de Alicante para estimar la varianza poblacional en función de la variable del local de residencia. En esta fase se cumplimentaron 6 encuestas, donde se observó el tiempo para ser contestadas, dudas, claridad de las cuestiones, posibles mejoras y el nivel de heterogeneidad $(\sigma)$.

Por el método del muestreo probabilístico se aplicó la ecuación (1) de una muestra (n) para una población finita, con una margen de error (E) de 10\%, un nivel de confianza de 95\%, a un nivel de heterogeneidad $(\sigma)$ de $75 \%$ para la populación, el muestreo recomendado debe tener un tamaño de muestra recomendado de 72 encuestas para una población de 35.727 personas. 
La encuesta ha sido contestada por 80 personas que han participado y todas consideradas en el estudio. Por el tratamiento estadístico de los datos se alcanza una 9,5\% de error muestral, considerado valido para el análisis de la muestra en lugar de lugar de la población completa. Para comprobar la veracidad de las respuestas, se ha establecido dos criterios: análisis de la marca temporal de las respuestas y tener contestado las preguntas obligatorias indicadas en la encuesta. En cuatro casos las encuestas han sido contestadas en intervalos de tiempo inferior de 10 minutos; después de hacer un análisis de los casos se han considerado válidas por tener respuestas muy distintas.

El cuestionario fue estructurado en 4 bloques: sección A: datos de clasificación; sección B: fenómenos naturales; sección C: paisaje y sección D: inundaciones. La encuesta tenía un total de 32 cuestiones (vid. Anexo I la encuesta aplicada en la Universidad de Alicante). La cumplimentación del cuestionario era opcional, para personas mayores de 18 años, anónimo y con preguntas cerradas.

Según la información estadística correspondiente al curso académico de la Universidad de Alicante de diciembre de $2011^{1}$, la comunidad académica de la Universidad de Alicante tenía: $79 \%$ de diplomaturas, licenciatura, $2^{\circ}$ ciclo y grados; $8 \%$ de posgrados y tercero ciclo; $6 \%$ de personal de administración y $7 \%$ de personal docente e investigador.

\section{DIVULGACIÓN DE LAS ENCUESTAS}

En la Universidad de Alicante se llevaron a cabo encuestas online entre los miembros de su comunidad universitaria para analizar cómo perciben los riesgos naturales. El cuestionario estaba disponible en una página web y fue enviado el enlace a todos los departamentos de la Universidad de Alicante. También se publicó una noticia, al respecto, en la página de Actualidad Universitaria de la web de la Universidad de Alicante (Figura 4). El cuestionario estuvo disponible entre los días 13 de noviembre y 13 de diciembre de 2012.

Figura 4. Divulgación de las encuestas en la Universidad de Alicante en noviembre de 2012.

\begin{tabular}{|c|c|}
\hline Españod | Volencid | Enclish | & 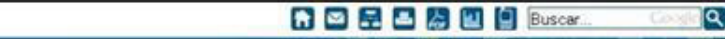 \\
\hline $\begin{array}{l}\text { Universitat d'Alacant } \\
\text { Universidad de Alicante }\end{array}$ & 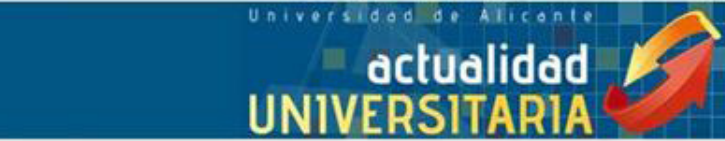 \\
\hline \multicolumn{2}{|l|}{ Directorio I } \\
\hline $\begin{array}{l}\text { - SALA DE PRENSA } \\
\text { - Unidad de Comuncadón } \\
\text { - Dossier de prensa } \\
\text { - Notas de prensa } \\
\text { : Ruedas de prensa } \\
\text { - Portal Audiovisual } \\
\text { - informadion y notias }\end{array}$ & 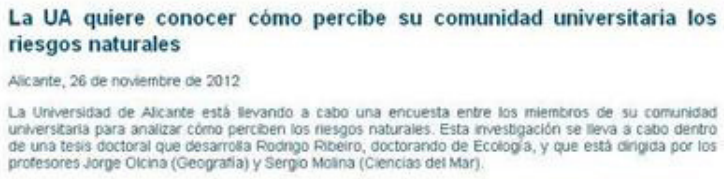 \\
\hline $\begin{array}{l}\text { - Siquenos en Facebook } \\
\text { Universidad de Alicante... } \\
\text { - ....y en Twitter Universidad }\end{array}$ & \multirow{3}{*}{ 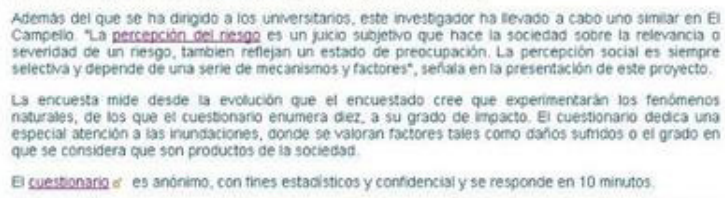 } \\
\hline \multirow{3}{*}{$\begin{array}{l}\text { - ACTUALIDAD UA } \\
\text { - Agenda general } \\
\text { "Portal de Ciencia y } \\
\text { Tecnologia } \\
\text { - Informasión cultural } \\
\text {-Deportes } \\
\text {-Boua }\end{array}$} & \\
\hline & \\
\hline & 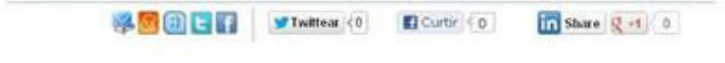 \\
\hline
\end{tabular}

\section{ANÁLISIS Y RESULTADOS}

El análisis de correlación de Pearson de las variables estudiadas puede definir el grado de relación con la variable de la percepción si viven en una zona amenazada por algún fenómeno natural. El análisis ha indicado que la experiencia con el riesgo es el factor de mayor relación con la percepción de riesgo. La Figura 5 presenta la variancia de la correlación de las variables, donde no hay una dependencia positiva de las variables cómo de género, tiempo que vive en la ciudad y edad.

1 Datos disponibles en la página web de la Universidad de Alicante. 〈http://utc.ua.es/es/datos/la-ua-en-cifras.html〉. [Consulta: 20/12/2012]. 
Figura 5. Correlación de la percepción con otras variables estudiadas.

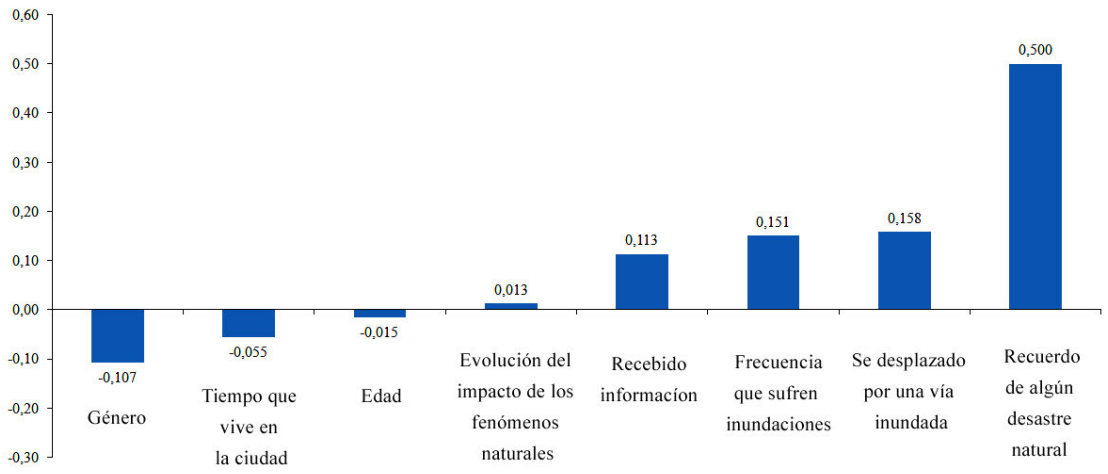

Los siguientes gráficos corresponden a la tabulación de las encuestas que han sido respondidas por los participantes. Los datos que se han extraído de estas encuestas, corresponden a aspectos de clasificación general, percepción de fenómenos naturales y percepción de las inundaciones.

\subsection{Datos de clasificación general}

En la muestra analizada se observó un equilibrio en la proporción de género. Con relación a la distribución del sexo de los encuestados en la Universidad de Alicante, 56\% son hombres y 44\% son mujeres. Con relación a la edad 21\% poseen entre 18 y 29 años, 63\% poseen entre 30 y 49 años y 16\% poseen 50 años o más. Con relación a lugar de residencia habitual: 43\% residen en Alicante, 23\% residen en San Vicente del Raspeig; 4\% residen en El Campello y 31\% en otra ciudad. En relación al tiempo que viven en la ciudad: 64\% 11 años o más; 15\% entre 6 y 10 años y $21 \%$ hasta 5 años.

\subsection{La percepción de fenómenos naturales}

En relación a la percepción de si creen que viven en un área amenazada por algún fenómeno natural: $68 \%$ creen que sí, $26 \%$ creen que no y $6 \%$ no saben o no contestan. Con relación a la pregunta se ha sucedido algún desastre natural que recuerde que haya dañado la comunidad donde vive el que contesta la encuesta: $66 \%$ ha contestado que sí 2 ; $31 \%$ que no y $3 \%$ que no saben o no contestan.

Con relación a la percepción del grado de daño causado por algún tipo de fenómeno natural, la distribución de respuestas muestra que 57\% de los encuestados tienen una percepción de daño entre el grado 6 y 7 (Figura 6). Con relación a la pregunta de cómo creen que será la evolución del impacto causado por fenómenos naturales: $58 \%$ creen que empeorará; $28 \%$ creen que seguirá igual; $8 \%$ no saben o no contestan y $8 \%$ que mejorará (Figura 7).

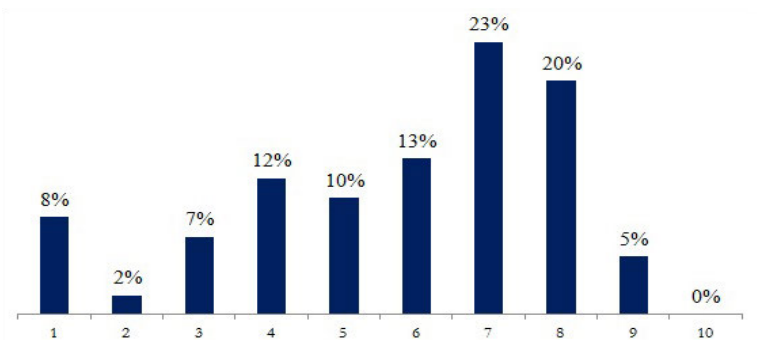

Figura 6. Percepción de daño causado por algún tipo de fenómeno natural (escala entre 1 y 10, siendo 10 es el grado más alto y 1 es el grado más bajo).

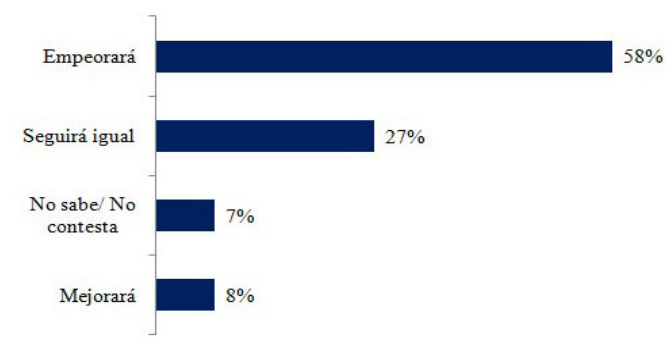

Figura 7. Percepción de la evolución del impacto causado por fenómenos naturales.

Respecto a los problemas ambientales que más preocupan los encuestados, los tres principales son: la desertificación, los incendios forestales y las inundaciones (Figura 8).

2 Se recuerda el último episodio ocurrido en el Campo de Alicante, el 30 de septiembre de 1997. 
Figura 8. Los problemas ambientales que más preocupan los encuestados (los encuestados podrían seleccionar más de una opción, por lo que los porcentajes pueden superar el 100\%).

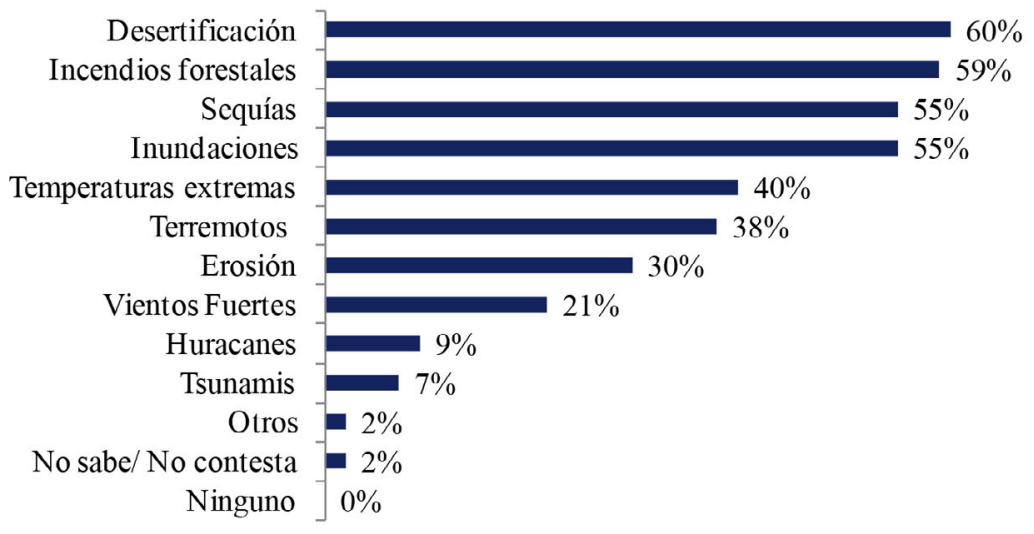

En relación a la percepción de las principales amenazas por fenómenos naturales que afectan o pueden afectar donde vive el encuestado, los tres problemas principales son: las inundaciones, las sequías y los incendios forestales (Figura 9).

Figura 9. Percepción de las principales amenazas por fenómenos naturales que afectan o pueden afectar donde vive el encuestado (los encuestados podrían seleccionar más de una opción, por lo que los porcentajes pueden superar el 100\%).

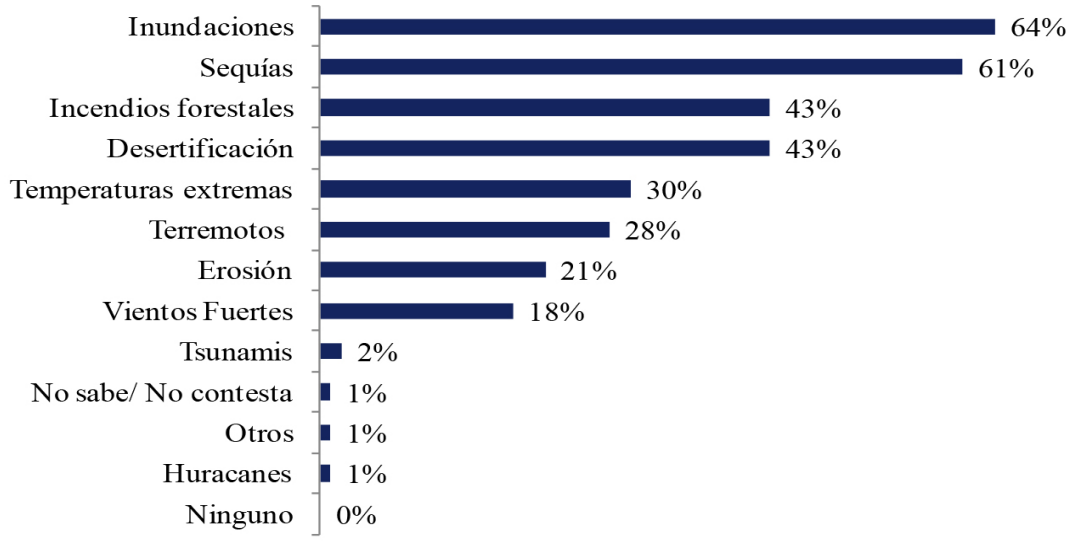

El análisis del grado cómo los problemas causados por fenómenos naturales le preocupan, la distribución de respuestas muestra que 60\% de los encuestados tienen una preocupación entre el grado 6 y 7 (Figura 10). Con relación a la percepción del grado de cómo creen que los impactos de los fenómenos naturales son producto de la sociedad, la distribución de respuestas muestra que $67 \%$ de los encuestados tienen una percepción entre el grado 7 y 9 (Figura 11).

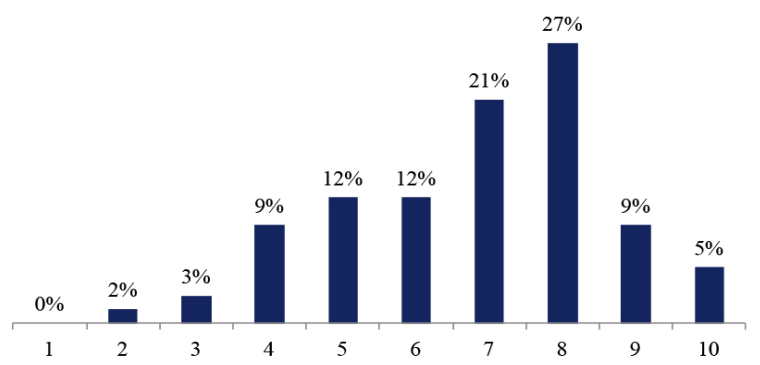

Figura 10. Preocupación con los problemas causados por fenómenos naturales (escala entre 1 y 10 , siendo 10 es el grado más alto y 1 es el grado más bajo).

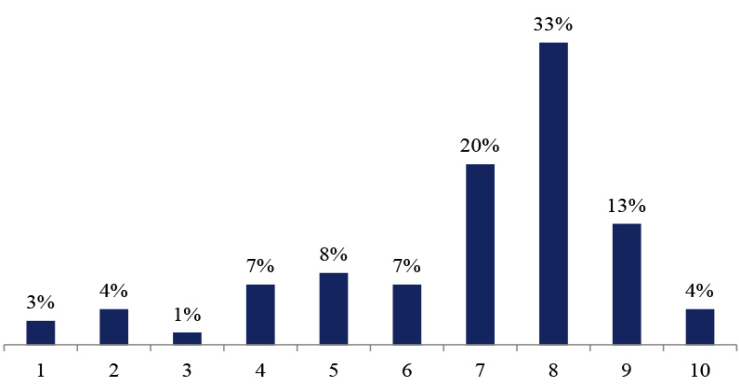

Figura 11. Percepción de como los impactos de los fenómenos naturales son producto de la sociedad (escala entre 1 y 10 , siendo 10 es el grado más alto y 1 es el grado más bajo). 
Por su parte, a la cuestión de si han recibido alguna vez información sobre riesgos naturales: 54\% han contestado que sí, $44 \%$ han contestado que no y $4 \%$ no contestan o no saben. Con relación a si han recibido alguna vez información sobre inundaciones: $44 \%$ han contestado que sí; $54 \%$ han contestado que no y $3 \%$ no contestan o no saben.

\subsection{La percepción de las inundaciones}

Del total de encuestados: $71 \%$ han contestado que ha tenido que desplazarse alguna vez por una vía inundada, $28 \%$ han contestado que no y $1 \%$ no contestan o no saben la respuesta. Con relación a la pregunta si han tenido informaciones a través de los medios de comunicación de inundaciones sucedidas en la zona que viven, $76 \%$ han contestado que sí, $19 \%$ han contestado que no y $5 \%$ no saben o no contestan la pregunta.

Con relación al grado de preocupación por las inundaciones donde viven los encuestados, la distribución de respuestas muestra que $61 \%$ de los encuestados tienen una preocupación entre el grado 6 y 8 (Figura 12). Respecto a la percepción del grado de los daños que han sufrido en el lugar donde viven los encuestados debido a las inundaciones, la distribución de respuestas muestra que $41 \%$ de los encuestados tienen una percepción entre el grado 7 y 8 (Figura 13).

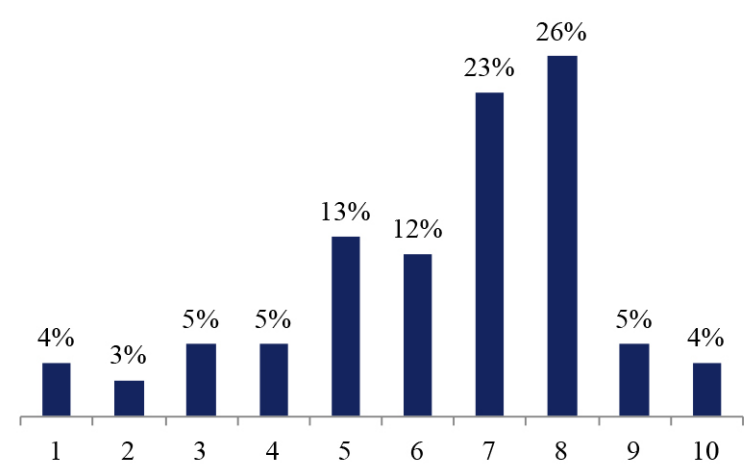

Figura 12. Grado de preocupación por las inundaciones donde viven los encuestados (escala entre 1 y 10 , siendo 10 es el grado más alto y 1 es el grado más bajo).

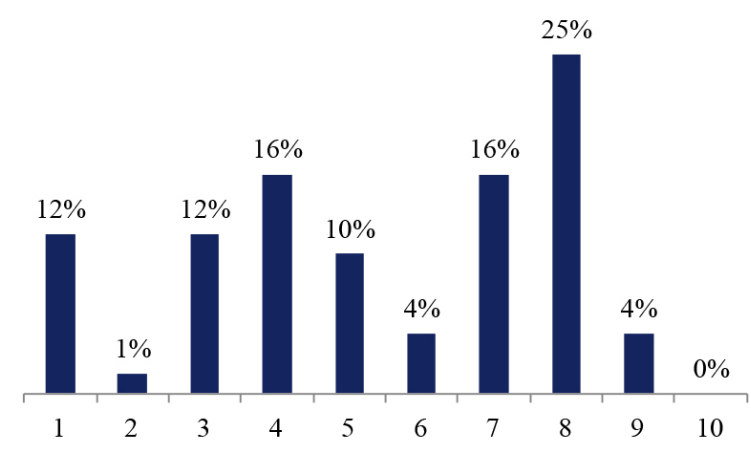

Figura 13. Percepción del grado de los daños que han sufrido en el lugar donde viven los encuestados debido las inundaciones (escala entre 1 y 10 , siendo 10 es el grado más alto y 1 es el grado más bajo).

En el caso de la percepción del grado que han sufrido con las inundaciones, la distribución de respuestas muestra que $42 \%$ de los encuestados tienen una percepción entre el grado 3 y 5 (Figura 14). Con relación a la percepción del grado que creen que las inundaciones son producto de la sociedad, la distribución de las respuestas muestra que 50\% de los encuestados tienen una percepción entre el grado 7 y 9 (Figura 15).
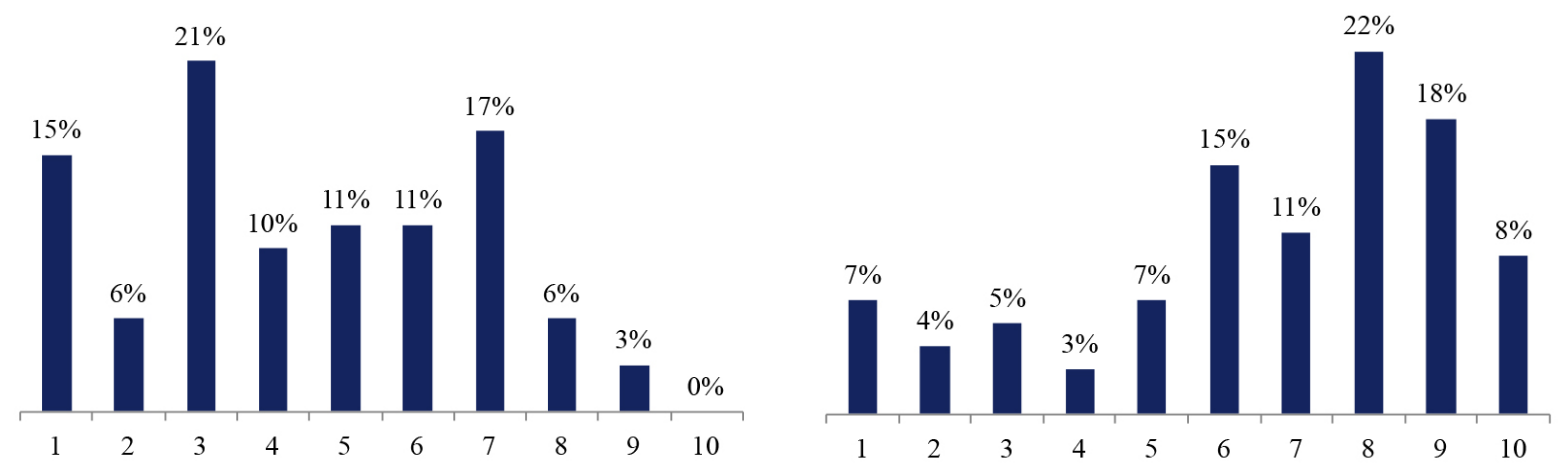

Figura 14. Percepción del grado que han sufrido con las inunda- Figura 15. Percepción del grado que creen que las inundaciones son ciones (escala entre 1 y 10, siendo 10 es el grado más alto y 1 es el producto de la sociedad (escala entre 1 y 10 , siendo 10 es el grado grado más bajo). más alto y 1 es el grado más bajo). 
Por último, respecto a la percepción de la frecuencia con que se sufren los impactos de las inundaciones, $48 \%$ han contestado entre 5 y 10 años, $16 \%$ han contestado 10 años o más, $16 \%$ han contestado no saben o no contestan, $10 \%$ han contestado que nunca han sufrido impacto de las inundaciones y $10 \%$ han contestado sufre impacto de las inundaciones cada año (Figura 16).

Figura 16. Percepción de la frecuencia sufren los impactos de las inundaciones.

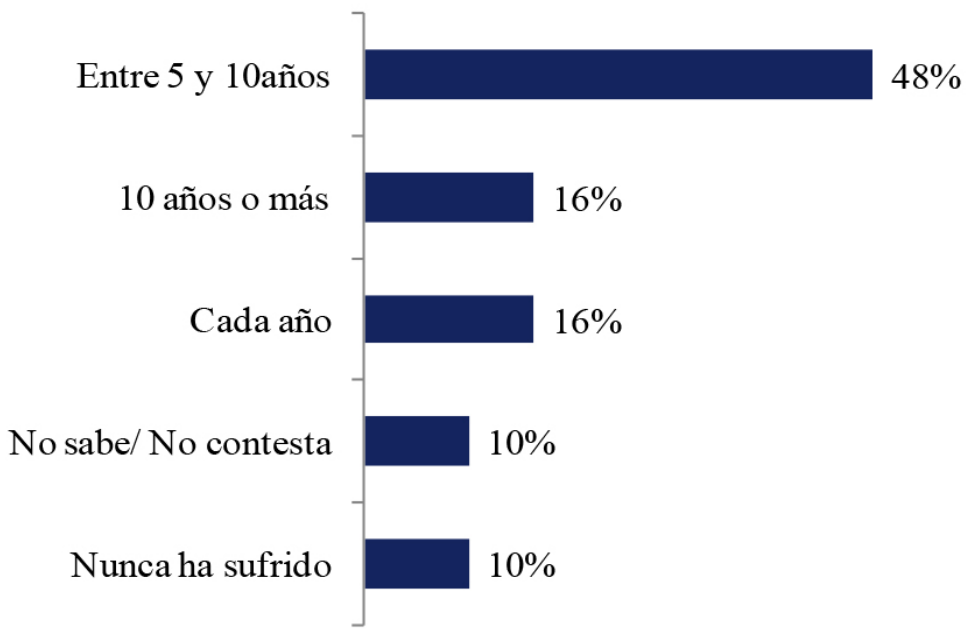

\section{CONCLUSIONES}

La realización de encuestas online es una metodología que aporta la posibilidad al encuestado de poder contestar en un momento que sea mejor para el interesado, pero también está sujeta a una confiabilidad distinta de encuestas impresas o por teléfono. Por haber informado en el inicio de la encuesta que se trata de un cuestionario anónimo no se ha establecido en los criterios de discriminación desde donde se han producido la misma. Se ha analizado la marca temporal de cada encuesta, con el objetivo de validar las encuestas.

La posibilidad de divulgación en la página web de la Universidad de Alicante ha sido un factor importante para hacer el trabajo en este centro universitario. Se observó un equilibrio en la proporción de género, con la mayoría (63\%) con edad entre 30 y 49 años y 66\% residen el Alicante o San Vicente del Raspeig. Los problemas ambientales que más preocupan los encuestados son: la desertificación, los incendios forestales y las inundaciones. En relación a la percepción del grado que han sufrido con las inundaciones, la distribución de respuestas muestra una tendencia decreciente para el grado más alto.

La percepción social de los riesgos naturales es producto de muchos factores como el resultado del universo social y grado de conocimiento de situaciones similares. Se ha observado en la muestra estudiada en el artículo que las personas que pasan por dificultades debido a efectos de fenómenos naturales, como por ejemplo las inundaciones, son más conscientes en relación a las acciones que debemos tomar respecto al medio ambiente. La experiencia con el riesgo, esto es, la vivencia de algún episodio extremo en fecha reciente, es el factor de mayor correlación con la percepción del riesgo, en la muestra estudiada. 


\section{ANEXO I. MODELO DE ENCUESTA APLICADA EN LA UNIVERSIDAD DE ALICANTE}

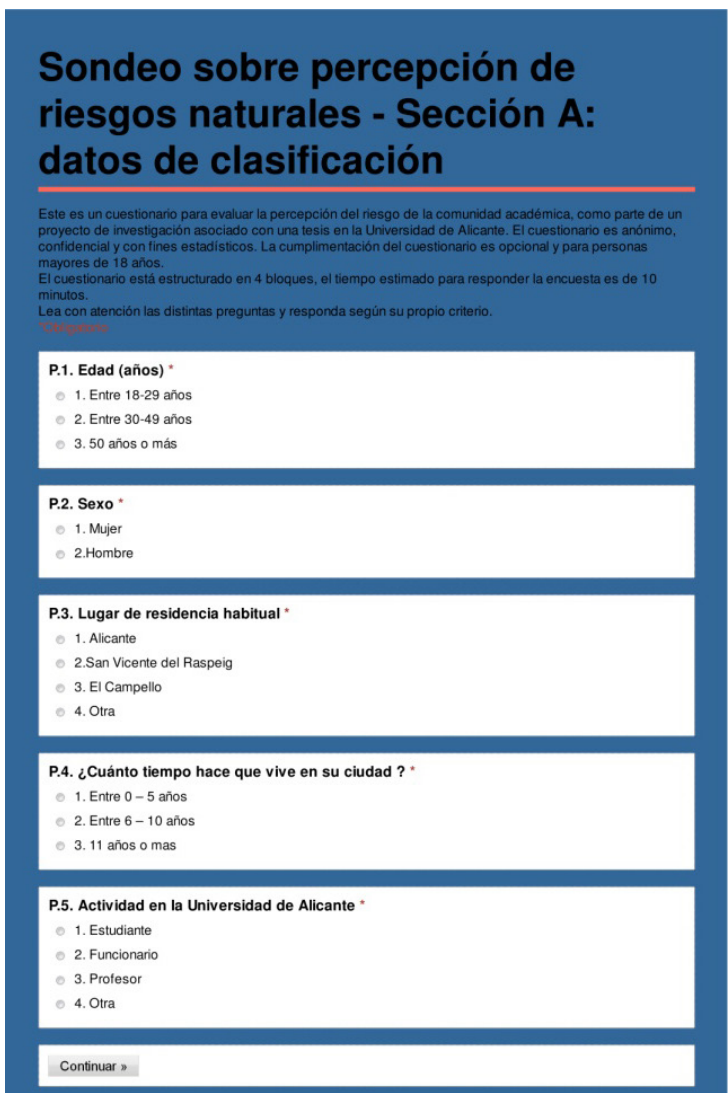

P.11. Indique en que grado los problemas causados por fenómenos naturales le preocupan, valore entre 1 y 10, (10 es el grado mas alto y 1 es el grado más bajo). Si "no (1)

$\begin{array}{llllllllll}1 & 2 & 3 & 4 & 5 & 6 & 7 & 8 & 9 & 10\end{array}$

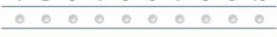

P.12. Señale cuáles son las principales amenazas por fenómenos naturales que afectan o pueden afectar donde usted vive

1. Temperaturas extremas

3. Terremotos

4. Incendios forestales

15. 4. Desertificación

5. Inundaciones

4. 6equias

4. Tsunamis

8. Huracanes

a 9. Erosión

10. Vientos Fuertes

11. Ninguno

412 Otros

E13. No sabe/ No contesta

P.13. Indique en que grado cree que los impactos de los fenómenos naturales son producto de la sociedad, valore entre 1 y 10 (10 es el grado mas alto y 1 es el grado más bajo). Si "no sabe/ no contesta" no marque nada.

$\begin{array}{llllllllll}1 & 2 & 3 & 4 & 5 & 6 & 7 & 8 & 9 & 10\end{array}$

$\begin{array}{llllllllllllllll}1 & 0 & 0 & 0 & 0 & 0 & 0 & 0 & 0 & 0 & 0\end{array}$

P. 14. ¿Ha recibido usted alguna vez información sobre riesgos naturales ¿

$01 . \mathrm{Si}$

3. No sabe/ No contesta

P.15. ¿ $\mathrm{Ha}$ recibido usted alguna vez información sobre inundaciones $¿ *$

- 1. Si

2. No

- 3. No sabe/ No contesta

*Atrás Continuar *
Sondeo sobre percepción de riesgos naturales Sección B: fenómenos naturales

P.6. ¿Crees que vive en una área amenazada por algún fenómeno natural? *

P.6. ¿Cre $1 . \mathrm{Si}$
$-2 . \mathrm{No}$

3. No sabe/ No contesta

P.7. ¿Ha sucedido algún desastre natural que recuerde que haya dañado la comunidad

1. si

2. No

3. No sabe/ No contesta

P.8. Indique el grado de daño causado por algún tipo de fenómeno natural, valore entre 1 sólo una opción (10 es el grado mas alto y 1 el grado más bajo)

$\begin{array}{llllllllll}1 & 2 & 3 & 4 & 5 & 6 & 7 & 8 & 9 & 10\end{array}$

$\begin{array}{lllllllllll}1 & 2 & 0 & 0 & 0 & 0 & 0 & 0 & 0 & 0 & 0\end{array}$

P.9. ¿Cuál cree que será la evolución del impacto de los fenómenos naturales? *

1. Empeorará

4. Mejorará

3. Seguirá igual

4. 4o sabe/ No contesta

P.10. Seleccione los problemas ambientales que mas le preocupan

v1. Temperaturas extremas

- 2. Terremotos

3. Incendios forestales

4. Desertificación

4. Inundaciones

6. Sequias

7. Tsunamis

8. Huracanes

8. Huracanes

(10.

10. Vientos Fuertes

11. Ninguno

12. Otros

13. No sabe/ No contesta

Sondeo sobre percepción de riesgos naturales

Sondeo sobre
Sección C: paisaje

P.16. ¿Cree que hay algún paisaje que sea representativo y caracteristico de donde vive?

01.5

2. No

3. No sabe/ No contesta

P.17. Indique cuáles de los siguientes elementos le resulta más relevante en el paisaje donde vive *

II. Ambiental

a 2. Económico

3. Historico

4. Estético

15. Social

6. Recreativo

7. Otros

D. Binguno

9. No sabe/ No contesta

P.18. Valore del 1 al 10 su preocupación por paisajes amenazados por fenómenos contesta" no marque nada.

$\begin{array}{llllllllll}1 & 2 & 3 & 4 & 5 & 6 & 7 & 8 & 9 & 10\end{array}$

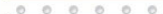

P.19. Señale a continuación los elementos que considere negativos, que cree que

1. Contaminación visual

- 2. Deterioro ambiental

3. Deterioro del patrimonio

4. Fragenter tentonia

4. B. Banalidad (sin personalidad propia)

6. Falta de integración con el entorno

a 7. Otros

8. Ningun

a. 9 . No sabe/ No contesta 
P.20. Por favor, valore del 1 al 10 los cambios que se ha notado en el paisaje debido a fenómenos naturales (donde 10 es el grado mas alto y 1 es el grado más bajo). Si "no

$\begin{array}{llllllllll}1 & 2 & 3 & 4 & 5 & 6 & 7 & 8 & 9 & 10\end{array}$

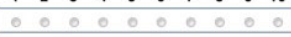

P.21. Por favor, valore del 1 al 10 los cambios que se ha notado en el paisaie debido a inundacio el paje debido a inundaciones (dorde

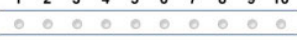

P.22. Valore del 1 al 10 su preocupación por paisajes amenazados por inundaciones (donde 10 es el grado mas alto y 1 es el grado más bajo). Si "no sabe/ no contesta" no marque nada.

$\begin{array}{llllllllll}1 & 2 & 3 & 4 & 5 & 6 & 7 & 8 & 9 & 10\end{array}$

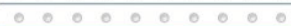

P.23. ¿Puede identificar en el paisaje donde vive daños o efectos como producto de las inundaciones? *

- $1 . \mathrm{Si}$

2 No

3. No sabe/ No contes:

P.24. Basándose en su experiencia, por favor, valore del 1 al 10 su preocupación a cerca P.24. Basandose en su experiencia, por favor, valore del 1 al 10 su preocupacion a cerca
del cambio del paisaje donde vive (donde 10 es el grado mas alto y 1 es el grado más del cambio del paisaje donde vive (donde 10 es el
bajo). Si "no sabe/ no contesta" no marque nada.

$\begin{array}{llllllllll}1 & 2 & 3 & 4 & 5 & 6 & 7 & 8 & 9 & 10\end{array}$

\begin{tabular}{llllllllll}
0 & 0 & 0 & 0 & 0 & 0 & 0 & 0 & 0 & 0 \\
\hline & 0 & 0 & 0 & 0
\end{tabular}

P.25. Indique en que medida identifica alguna degradación ambiental del paisaje donde P.25. value del 1 al 10 (donde 10 es el grado mas alto $y 1$ es el grado más baje) donde sabe/ no contesta" no marque nada.

$\begin{array}{llllllllll}1 & 2 & 3 & 4 & 5 & 6 & 7 & 8 & 9 & 10\end{array}$

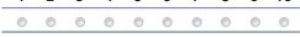

«Atrás Continuar \#

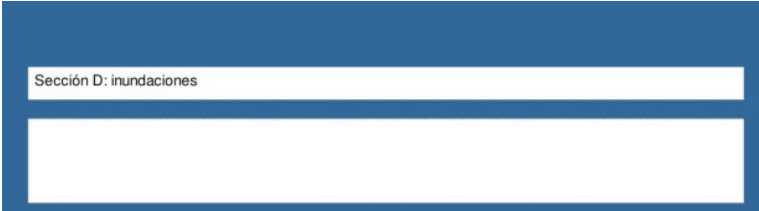

P.26. ¿Alguna vez ha tenido que desplazarse por una via inundada donde usted vive? * 1. Si

1. $1 . \mathrm{Si}$

3. No sabe/ No contesta

P.27. ¿Ha tenido informaciones a través de los medios de comunicación de inundaciones en la zona que usted vive?

- 1. Si

2. No

3. No sabe/ No contesta

P.28. Basándose en su experiencia, por favor, valore del 1 al 10 su preocupación por las daciones donde vive (10 es el grado mas alto y 1 es el grado más bajo). Si "no sabe no contesta" no marque nada.

$\begin{array}{llllllllll}1 & 2 & 3 & 4 & 5 & 6 & 7 & 8 & 9 & 10\end{array}$

P.29. Valores entre 1 y 10 los daños ha sufrido el lugar donde usted vive a causa de las inundaciones, valore entre 1 y 10, marque solo una opción (10 es el grado mas alto y

$\begin{array}{llllllllll}1 & 2 & 3 & 4 & 5 & 6 & 7 & 8 & 9 & 10\end{array}$

$\begin{array}{llllllllllllllllll}1 & 0 & 0 & 0 & 0 & 0 & 0 & 0 & 0 & 0 & 0\end{array}$

P.30. Indique en que grado usted ha sufrido con las inundaciones, valore entre 1 y 10 , marque sólo una opción (10 es el grado mas alto y 1 es el grado más bajo). Si "no sabe

$\begin{array}{llllllllll}1 & 2 & 3 & 4 & 5 & 6 & 7 & 8 & 9 & 10\end{array}$

$\begin{array}{lllllllllllllllllll}1 & 0 & 0 & 0 & 0 & 0 & 0 & 0 & 0 & 0 & 0\end{array}$

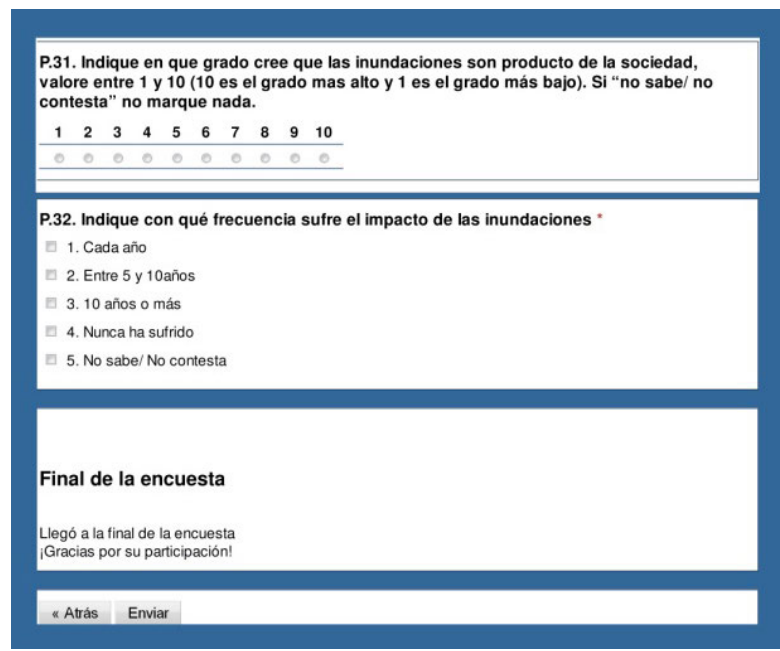

\section{BIBLIOGRAFÍA}

ACOSTA, V.G. (2005): "El riesgo como construcción social y la construcción social de riesgos" en Desacatos del Centro de Investigaciones y Estudios Superiores en Antropología Social. México, nº 019, pp. 11-24. ISBN 1405-9274.

ALMEIDA, A.B. (2004): "O conceito de risco socialmente aceitável como componente critico de uma gestão de risco aplicada aos recursos hídricos", en Actas del $7^{\circ}$ Congreso de la Agua, Asociación Portuguesa de Recursos Hídricos (APRH), Lisboa, 14 pp.

COElHO, C.O.A., VALENTE, S.M., PINHO, L.D., CARVAlHO, T.M., FERREIRA, A.D. y FIGUEIREDO, E.M. (2004): "A Percepção das Alterações Climáticas e do Risco da Cheia", en Actas del $7^{\circ}$ Congresso de Asociación Portuguesa de Recursos Hídricos (APRH), 13 pp. 
COELHO, C.D.B. (2005): Riscos de Exposição de Frentes Urbanas para Diferentes Intervenções de Defesa Costeira. Tese de Doctorado de la Universidad de Aveiro, 404 pp.

JUAN PÉREZ, J.I. (2007): Manejo del ambiente y riesgos ambientales en la región fresera del Estado de México. 162 pp. ISBN 978.84.690.6921-9.

OLCINA CANTOS, J. (2005): "La prensa como fuente para y estudio de los tiempos y climas" en Revista de historia moderna $\mathrm{n}^{\circ} 23$, pp. 185-232. ISSN 0212-5862.

WEINGART, P. (2007): Risiko aus soziologischer perpektive. Debatte Helft 6 Berlin - Brandenburgische Akedemie de Wissenchaften, Risiko, 19 pp. ISBN 978.3.939818-09-0. 